\title{
Transistor Oscillator and Amplifier Grids
}

\author{
ROBERT M. WEIKLE II, MEMBER, IEEE, MOONIL KIM, STUDENT MEMBER, IEEE, JONATHAN B. \\ HACKER, STUDENT MEMBER, IEEE, MICHAEL P. DE LISIO, STUDENT MEMBER, IEEE, ZOYA B. \\ POPOVIĆ, MEMBER, IEEE, AND DAVID B. RUTLEDGE, SENIOR MEMBER, IEEE
}

Invited Paper

In this paper, we review recent developments in quasi-optical power combining. In particular, we examine planar periodic grids and their use as quasi-optical active components. A variety of grids used for the generation and amplification of electromagnetic radiation have been investigated. Although quasi-optical techniques are applicable to a large variety of solid-state devices, special attention is given to transistors. Transistors are attractive because they can be used as either amplifiers or oscillators. Experimental results for MESFET bar-grid and planar grid oscillators are presented. In addition, we discuss a MESFET grid amplifier that receives only vertically polarized waves at the input and radiates horizontally polarized waves at the output. An advantage of these planar grids is they can be scaled for operation at millimeter-and submillimeter-wave frequencies. By using modern IC fabrication technology, planar grid ascillators and amplifiers containing thou sands of devices can be built, thereby realizing an efficient means for large-scale power combining.

\section{INTRODUCTION}

Millimeter- and submillimeter-wave systems continue to be a subject of growing interest. The applications involving this portion of the electromagnetic spectrum cover a broad range of scientific disciplines, varying from the measurement of electron densities in tokamak plasmas [1] to studying emission spectra of distant celestial bodies [2]. Millimeter waves correspond to the frequencies between $30 \mathrm{GHz}$ and $300 \mathrm{GHz}$ and the submillimeter-wave range is regarded as the region between $300 \mathrm{GHz}$ and $3 \mathrm{THz}$. The shorter wavelengths at these frequencies allow the use of smaller and lighter components than for microwave

Manuscript received October 4, 1991; revised February 26, 1992. This research was supported by the Army Research Office, the Northrop Corporation, and a fellowship from the Rockwell International Trust. J. Hacker holds an NSERC Fellowship from Canada and M. De Lisio holds an NSF Fellowship.

R. M. Weikle II was with the Division of Engineering and Applied Science, California Institute of Technology, Pasadena, CA 91125. He is now with the Department of Applied Electron Physics, Chalmers is now with the Department of Applied Bniversity of Technology, Göteborg, Sweden.

M. Kim, J. B. Hacker, M. P. De Lisio, and D. B. Rutledge are with the Division of Engineering and Applied Science, California Institute of Technology, Pasadena, CA 91125.

Z. B. Popovic is with the Department of Electrical and Computer Engineering, University of Colorado, Boulder, CO 80309.

IEEE Log Number 9205761. systems. This is important in military and space-borne applications where size and weight are a prime concern. In addition, the atmospheric attenuation of millimeter and submillimeter waves is relatively low compared with infrared and optical wavelengths [3], particularly in the transmission windows that lie between water vapor and oxygen absorption peaks. This property can be exploited to build radars and cameras that penetrate clouds, smoke, and haze. Other commonly cited advantages of millimeterand submillimeter-wave components over their microwave counterparts include broader bandwidths and higher resolution for radars and imaging systems.

The lack of reliable, inexpensive high-power sources, however, has been a persistent obstacle in the development of millimeter- and submillimeter-wave systems. The first devices to produce radiation in this part of the spectrum were electron tubes. Today, electron tube devices such as klystrons and crossed-field amplifiers (CFA's) are widely available and can produce several kilowatts of power in the microwave and lower millimeter-wave range [4], [5]. Traveling-wave tubes are capable of better than $100 \mathrm{~W}$ at $100 \mathrm{GHz}$ [6]. In the far infrared and submillimeterwave range, optically pumped FIR lasers have achieved several megawatts of pulsed power [7]. Nevertheless, in many circumstances, the size, weight, and required highvoltage power supplies of these devices often limit their usefulness.

For most low and medium power applications, electron tube sources have been replaced by solid-state devices. Compared with tube sources, solid-state devices are small, lightweight, inexpensive, and require small to moderate voltages. At present, IMPATT's - the most powerful millimeter-wave solid-state sources - can produce several watts of power at $100 \mathrm{GHz}$. Silicon IMPATT's have produced useful power up to $300 \mathrm{GHz}$ [8]. A major drawback of IMPATT's, however, is the high noise level arising from the avalanche multiplication process. Gunn diodes have better noise performance but only generate a few hundred milliwatts at $100 \mathrm{GHz}$ [9]. Other two-terminal solid-state devices used to produce millimeter- and submillimeter-

0018-9219/92\$03.00 CC 1992 IEEE 
wave radiation include quantum well oscillators [10] and Josephson junctions [11], although the power levels are extremely small. An additional drawback of two-terminal solid-state sources is low dc-to-RF conversion efficiency. Better efficiencies can be realized with transistors. Pseudomorphic HEMT's have demonstrated output powers near 60 $\mathrm{mW}$ at $94 \mathrm{GHz}$ [12]. HEMT's with $0.15 \mu \mathrm{m}$ gate lengths have shown cutoff frequencies of over $170 \mathrm{GHz}$ with $f_{\max }$ near $350 \mathrm{GHz}$ [13]. Heterojunction bipolar transistors (HBT's) also have the potential to provide reasonable power levels at millimeter-wave frequencies without the need for submicron lithography. An HBT with emitter area of 80 $\mu \mathrm{m}^{2}$ has shown $15 \mathrm{~dB}$ of gain with output power of 16 $\mathrm{dBm}$ at $35 \mathrm{GHz}$ [14].

In an attempt to overcome the limited power output of solid-state devices, a variety of power-combining methods have been developed. A good review of these methods has been given by Chang and Sun for millimeter-wave frequencies [15]. Many of these techniques are based on scaled-down microwave circuits and involve resonant cavities [16] or hybrids. Resonant cavity combiners allow good isolation between the active devices and have been used up to $300 \mathrm{GHz}$. Hybrid power combiners, which often rely on external injection locking to synchronize the sources, have been used up to $140 \mathrm{GHz}$. These approaches have a number of disadvantages. To prevent moding problems, the size of the waveguide cavities must be scaled down at higher frequencies. This makes circuit fabrication more difficult. Resistive losses in the waveguide walls, which reduce power-combining efficiency, become more severe at millimeter and submillimeter frequencies. In addition, combiners based on resonant cavities and hybrids can accommodate a limited number of devices, making largescale power combining impractical.

\section{QuASI-OPTICAL POWER COMBININC}

An approach which overcomes the limitations of power combiners based on scaled-down microwave systems involves combining the output powers of many devices in free space. Mink suggested using an array of millimeter-wave devices placed in an optical resonator as a means of largescale power combining [17]. While it is unlikely that solidstate power combiners will replace high-power electron tube sources, there is great potential for improvement in output power and combining efficiency by using quasioptical techniques. Because the power is combined in free space, losses associated with waveguide walls and feed networks are eliminated. The power can be distributed over a larger number of devices than in a waveguide cavity because the quasi-optical resonator can be many wavelengths across. An external injection-locking signal is unnecessary because synchronization of the sources is accomplished by mutual coupling through the modes of the resonator.

Several types of quasi-optical power combiners have been demonstrated over the past few years. Wandinger and Nalbandian combined the outputs of two Gunn diodes at
$60 \mathrm{GHz}$ using tapered dielectric rod antennas coupled to a Gaussian resonator [18]. Many designs have used microstrip radiators which are synchronized with feedback or an external signal. Stephan et al. investigated the coupling between open resonators and microstrip circuits at $10 \mathrm{GHz}$ [19]. The microstrip ground plane and a spherical reflector formed the Gaussian cavity. Using this configuration, the output powers of two Gunn diodes were combined in free space [20]. A different approach involves an array of weakly coupled patch antenna elements [21]. This method is similar to classic antenna arrays; each patch antenna is a free-running oscillator containing an active device [22]. The patch elements, which may contain either Gunn diodes or MESFET's, are synchronized using separate de bias to each device. A dielectric slab placed above the array also facilitates locking. With this scheme, a 16-element MESFET array operating at $8 \mathrm{GHz}$ produced $184 \mathrm{~mW}$ of power with a dc-to-RF efficiency of $25 \%$. Linear arrays of patch antennas have also been used to combine the outputs of Gunn diodes at the second harmonic $(18 \mathrm{GHz})$ [23]. More recently, a two-sided microstrip configuration has been developed that permits isolation between an external locking signal and the array output [24].

A different approach to quasi-optical power combining is based on integrating solid-state devices directly into a periodic grid. Grid arrays have long been important components for infrared and millimeter-wave applications. Conductive meshes can be used as quasi-optical filters, beam splitters, and output couplers for lasers [25]-[27]. By integrating microbolometers and Schottky diodes into these grids, various investigators have demonstrated their use as multimode detectors, grid phase shifters, and quasi-optical multipliers [28]-[30].

\section{GRID OSCILLATORS}

Grid oscillators are periodic arrays embedded with active solid-state devices. The grid is placed in a Fabry-Perot resonator to provide the feedback necessary for oscillation. This is illustrated in Fig. 1. Two important features distinguish grid oscillators from most quasi-optical power combiners built from microstrip circuits. First, grid oscillators do not necessarily have a ground plane and, as a result, do not rely on the interaction of microstrip modes with free-space radiation. Second, microstrip-based power combiners tend to be a collection of individual free-running oscillators that are weakly coupled. Thus, the operating frequency depends primarily on the behavior of the individual oscillators. In contrast, the elements making up an oscillator grid are not themselves free-running oscillators. Mutual interaction of all the devices in the grid is necessary for oscillation to occur. Consequently, the oscillation frequency and the output power are strongly affected by the device spacing and the grid configuration. Each device in the array is presented with an embedding impedance which is a function of the grid structure. This embedding impedance, together with the device impedance, determines the grid's overall behavior as an oscillator. 


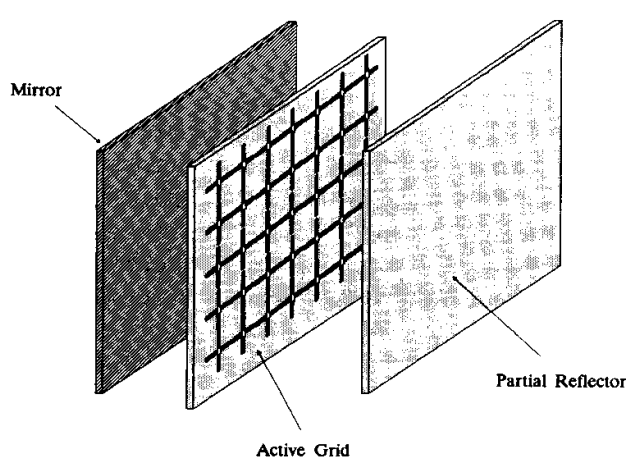

Fig. 1. Schematic of a grid oscillator. Active devices are embedded in a two-dimensional periodic array supported by a dielectric substrate. The grid array is placed in a Fabry-Perot cavity that couples the devices together.

In principle, any solid-state device can be used in an oscillator grid. Although readily amenable to planar integration, two-terminal devices generally have poor dcto-RF efficiencies and can prove difficult to synchronize. Transistors, on the other hand, have respectable dc-to-RF conversion efficiencies and a separate control terminal. This allows the devices to be more easily stabilized, permitting oscillation to be controlled through an appropriately designed feedback circuit

A variety of transistor grid configurations have been investigated. The first transistor oscillator grid, demonstrated by Popović et al. [31] in 1988, is shown in Fig. 2(a). The array is fabricated on a dielectric substrate (Duroid, Rogers Corporation) with $\epsilon_{T}=10.5$ and a thickness of $2.35 \mathrm{~mm}$. Packaged MESFET's (Fujitsu FSC11LF) are soldered into the grid. The vertical metal lines, which are connected to the transistor drain and gate terminals, are parallel to the radiated electric field. Horizontal metal leads running across the grid are used for dc biasing. The back side of the substrate is metallized and serves as both a mirror and ground for the MESFET source leads. Figure 2(b) shows details of the grid configuration. The device spacing is $\mathbf{1 3}$ $\mathrm{mm}$. The gate, which is connected to a $5 \mathrm{~mm}$ long inductive strip, is not dc biased. When $4 \mathrm{~V}$ is applied to the drain, the grid oscillates at $9.7 \mathrm{GHz}$. Figure 3 shows the spectrum. The metallized back side of the substrate and a planar dielectric slab ( $\epsilon_{r}=10.5$ and thickness of $2.5 \mathrm{~mm}$ ) placed in front of the grid form a Fabry-Perot resonator. Varying the distance of the front dielectric slab tunes the frequency about $1 \%$ and the output power by nearly $10 \%$. The total radiated power, calculated by measuring the far-field radiation pattern, is $464 \mathrm{~mW}$. This corresponds to an effective radiated power (ERP) of $20.7 \mathrm{~W}$ and a dc-to-RF conversion efficiency of about $15 \%$. The maximum ERP obtained from the grid was 37 watts.

An attractive feature of planar grid arrays is they can be modeled with relatively simple transmission-line circuits. Fig. 4 shows an example. Energy radiated from the grid is modeled as a wave propagating along a transmission

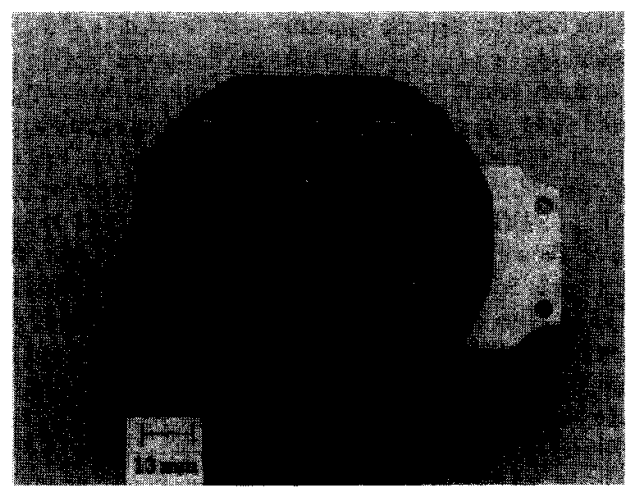

(a)

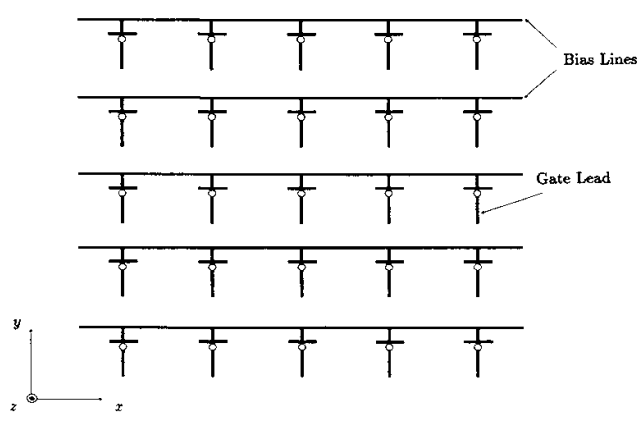

(b)

Fig. 2. (a) Photograph of a 25-element MESFET grid oscillator. The back side of the substrate is metallized and a dielectric slab is placed in front of the grid to form a Fabry-Perot cavity. (b) Schematic of the grid configuration. The MESFET drain and gate are connected to the vertical leads. The source leads run through the substrate and are soldered to the ground plane [31].

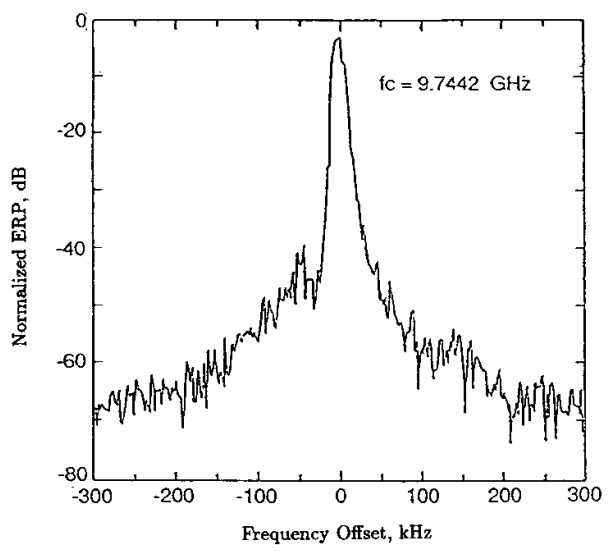

Fig. 3. Spectrum of the 25-element MESFET grid [31].

line. The characteristic impedance of the transmission line corresponds to the TEM impedance for a wave traveling

PROCEEDINGS OF THE IEEE, VOL. 80, NO. 11, NOVEMBER 1992 


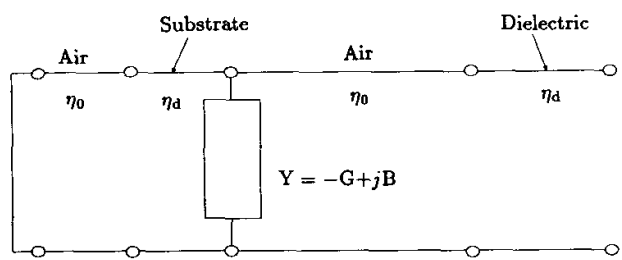

Fig. 4. Transmission-line model for a planar grid. Radiated power isrepresented as a wave traveling along a transmission line.

in that particular dielectric medium. Thus, for the grid of Fig. 2(a), free space is represented with a $377 \Omega$ transmission line and the dielectric slabs are modeled with $116 \Omega$ lines. A short circuit models the mirror behind the grid. A shunt admittance, $Y$, represents the devices and the embedding impedance. This admittance is a function of the grid configuration as well as the impedance of the embedded devices. For a grid containing active solid-state devices, the real part of the admittance is negative.

\section{BAR-GRID OSCILLATORS}

An alternative quasi-optical grid configuration is shown in Fig. 5. The grid consists of an array of metal bars on which packaged devices are mounted. This structure has been used to combine the output powers of both transistors [32] and Gunn diodes [33]. A mirror placed behind the grid couples the devices together and is also used for reactive tuning. The metal bars, which are used to provide dc bias to the devices, make an excellent heat sink. For convenience, the devices in adjacent rows share dc biasing. This arrangement minimizes the number of biasing connections. It also gives the grid a symmetric structure that can be exploited to determine the grid's embedding impedance.

A transmission-line model similar to that shown in Fig. 4 is used to describe the bar grid. Each device in the grid is viewed as occupying a unit cell which is defined by symmetry. If the devices in the grid are identical and all are oscillating in phase, the electric and magnetic fields must satisfy symmetry-imposed boundary conditions along the edges of the unit cell. In effect, the entire grid is reduced to an equivalent waveguide representation as illustrated in Fig. 5(b). The equivalent waveguide has electric walls on the top and bottom and magnetic walls (where the tangential magnetic field vanishes) on the sides. A device placed in the grid is viewed as a source which excites the equivalent waveguide. By finding the impedances present at the terminals of a device in the unit cell, the behavior of the grid can be predicted. Details of the analysis have already been presented and it is unnecessary to repeat them here [32]. A transmission-line model representing the MESFET bar-grid oscillator is shown in Fig. 6. The drain and gate leads excite different waveguides which are formed by the metal bars. These waveguides are modeled with two sections of transmission line with characteristic impedance

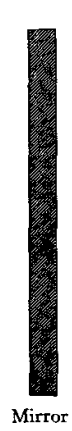

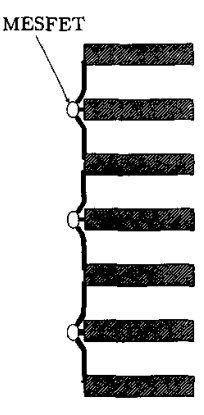

Mirror

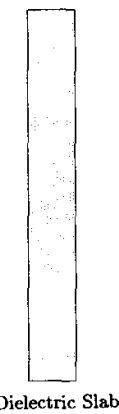

Dielectric Slab (a)

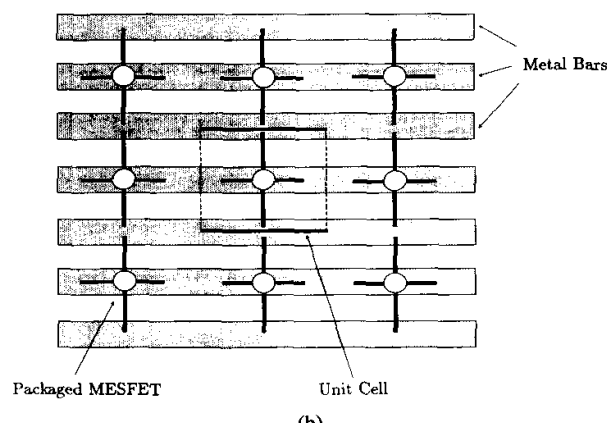

(b)

Fig. 5. (a) Side view of the MESFET bar-grid oscillator. (b) Front view of the bar grid. Adjacent rows of devices share bias. The unit cell is shown with solid lines to indicate electric walls and dashed lines to represent magnetic walls.

$Z_{\mathrm{TEM}}$. The discontinuity at the edges of the metal bars produce evanescent capacitive modes and is represented with lumped capacitors, $C$ and $C_{m} . C_{m}$ is the mutual capacitance arising from fields of one waveguide coupling to the fields of the other waveguide. Currents on the drain and gate leads generate evanescent inductive modes which we model with lumped inductors. There is also a mutual inductance, $M$, describing the coupling of the magnetic fields between the metal-bar waveguides.

A grid containing 36 MESFET's mounted on metal bars produced $220 \mathrm{~mW}$ of power at $3 \mathrm{GHz}$. The grid period is 10 $\mathrm{mm}\left(0.1 \lambda_{0}\right)$. The measured dc-to-RF conversion efficiency was $22 \%$. Grid directivity, measured from the far-field radiation pattern, was $11.3 \mathrm{~dB}$. Moving the position of the back-short tunes the operating frequency of the grid over a $300 \mathrm{MHz}$ bandwidth. In addition, the frequency can be varied by changing the bias to the gate leads $(250 \mathrm{MHz} / \mathrm{V})$. Gate bias, however, has little effect on the amplitude of the radiated signal, indicating that this property can be used to frequency modulate the grid output. Fig. 7 shows the output spectrum of the bar grid when a $5 \mathrm{MHz}$ ac signal is superimposed over the gate bias. The FM Bessel function coefficients [35] are shown for comparison. 


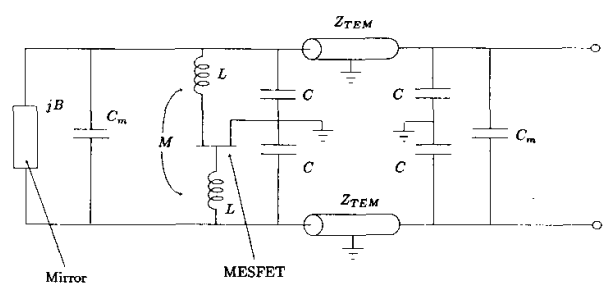

Fig. 6. Transmission-line model of the MESFET bar-grid oscillator. The MESFET is added to the model using its small-signal equivalent circuit.

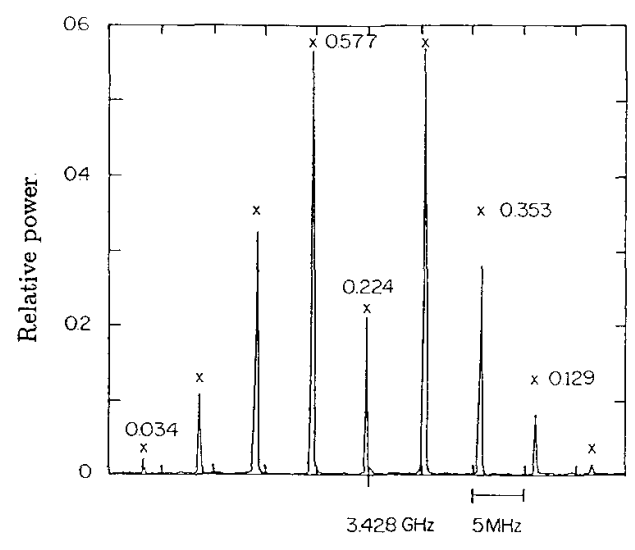

Frequency

Fig. 7. Measured bar-grid oscillator FM spectrum. The scale is linear in power and the Bessel function coefficients are shown with $x$ 's [32]

\section{Planar-Grid OSCILlators}

The transistor grids discussed thus far operate at microwave frequencies. To extend the operating frequencies to the millimeter- and submillimeter-wave region, highfrequency devices need to be integrated into the grid. Packaged devices are unsuitable because of the associated parasitics. In addition, the devices in the grid need to be placed closer together at higher frequencies. Thus, millimeter- and submillimeter-wave grids are only feasible if monolithic fabrication techniques are used. For this reason, planar grid configurations are particularly important.

Two types of planar grids have been investigated to date [36], [37]. The grids, which have the same basic structure, differ by the manner in which the MESFET's are connected. A schematic of the planar grid is shown in Fig. 8. Like the bar grid, vertical leads couple to the radiated field and horizontal leads are used for dc biasing. Two transistor terminals are connected to the vertical leads while the third is connected to the horizontal bias line. Initial work with the planar grid structure utilized packaged MESFET's (Fujitsu FSC11LF). Because of the physical layout of the device package, the grid was restricted to the vertical drain-gate configuration illustrated in Fig. 9(a). Subsequent investiga-

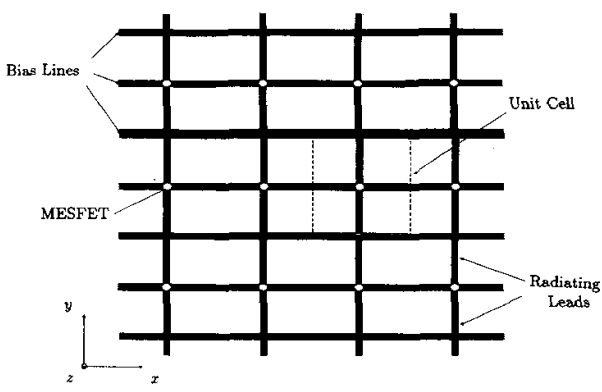

Fig. 8. Physical layout of the planar MESFET grid oscillator. Adjacent rows of devices share bias lines. The unit cell equivalent waveguide is shown with solid lines for electric walls and dashed lines for magnetic walls.

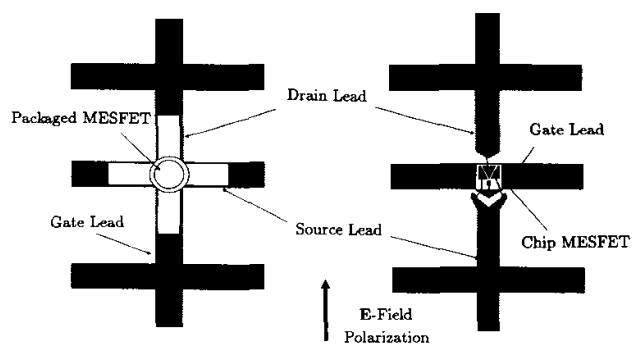

(a)

(b)

Fig. 9. (a) Unit cell for a vertical drain-gate grid which uses packaged devices. (b) Unit cell for a gate-feedback grid oscillator with chip MESFET's. The gate lead, which runs horizontally, is capacitively coupled to the incident electric field [37].

tions involved chip MESFET's (Fujitsu FSC11X) which were connected in a vertical drain-source configuration (Fig. 9(b)).

To find a grid equivalent circuit model, symmetry is exploited to define a unit cell equivalent waveguide. An EMF analysis of the unit cell (similar to that used by Eisenhart and Khan for a post in a waveguide [34]) leads to the transmission-line model of Fig. 10. The terminals labeled 1 and 2 represent connections to the vertical leads of the grid. The center terminal labeled 3 represents the horizontal lead. Free space is modeled with a $377 \Omega$ transmission line which is scaled by the aspect ratio $(b / a)$ of the unit cell. A lumped inductor, $L$, accounts for the inductance of the vertical leads. Currents in the horizontal leads are orthogonal to the radiated field and thus generate nonpropagating (evanescent) modes. These modes are represented with the lumped reactive elements, $C_{m}$ and $L_{m}$. Coupling between the radiated field and currents in the various transistor leads is described by a center-tapped transformer.

Strictly speaking, a transmission-line model derived using an equivalent waveguide is only valid for infinite grids. As a result, the applicability of the model is questionable with small grids because the equivalent waveguide boundary

PROCEEDINGS OF THE IEEE, VOL. 80, NO. 11, NOVEMBER 1992 


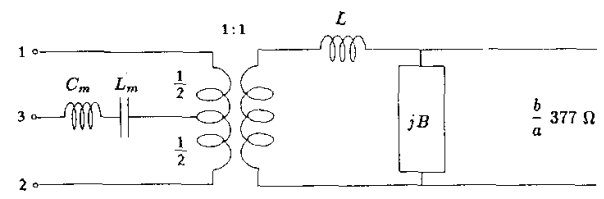

Fig. 10. Transmission-line model for the planar MESFET grid. The mirror behind the grid is represented with a shunt susceptance $j B$ [37].

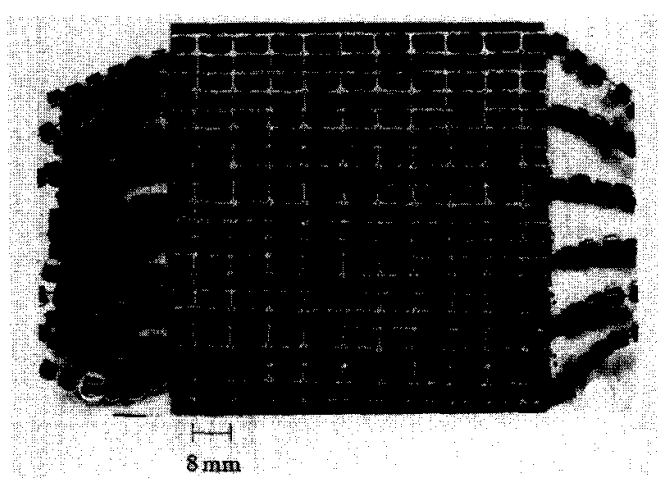

Fig. 11. Photograph of the 100-element MESFET grid. Ferrite beads placed in the bias lines suppress low-frequency oscillation. The vertical leads are parallel to the radiated electric field [36].

conditions do not hold at the grid edges. It may be possible, however, to terminate the grid edges in a way that simulates the equivalent waveguide boundary conditions. Electric walls at the top and bottom of the grid can be approximated with vertical $\lambda / 4$ open-circuited stubs. This should prevent currents there from abruptly dropping to zero. In addition, RF chokes or inductive leads can be used at the sides of the grid to create large impedances, thus simulating magnetic walls. Using edge terminations such as these, we have found the EMF models to be in reasonably good agreement with measurement for grids containing as few as 16 elements.

MESFET arrays based on the vertical drain-gate configuration of Fig. 9(a) are called source-feedback grids. This terminology arises because the vertical leads (drain and gate) couple directly to the radiated field, and part of this radiated field is capacitively coupled to the horizontal transistor lead (the source) through the grid embedding circuit. A 100 -element grid based on the unit cell of Fig. 9(a) is shown in Fig. 11 [36]. The grid is built on a $0.5 \mathrm{~mm}$ thick substrate $\left(\epsilon_{r}=2.2\right)$ which lies on top of a second substrate ( $2.5 \mathrm{~mm}$ thick and $\left.\epsilon_{r}=10.5\right)$. Devices in the grid are spaced $8 \mathrm{~mm}$ apart. The grid, which oscillates at $5 \mathrm{GHz}$, produces $600 \mathrm{~mW}$ of power with a dc-to-RF efficiency of $20 \%$. A planar mirror behind the grid is used to tune the frequency and output power (Fig. 12).

A disadvantage of the common-source grid is the radiating gate lead. Because the gate strongly couples to the radiated field, the grid tends to oscillate at lower frequencies where the devices have high gain. The common-gate grid

WEIKLE e al: TRANSISTOR OSCILLATOR AND AMPLIFIER GRIDS

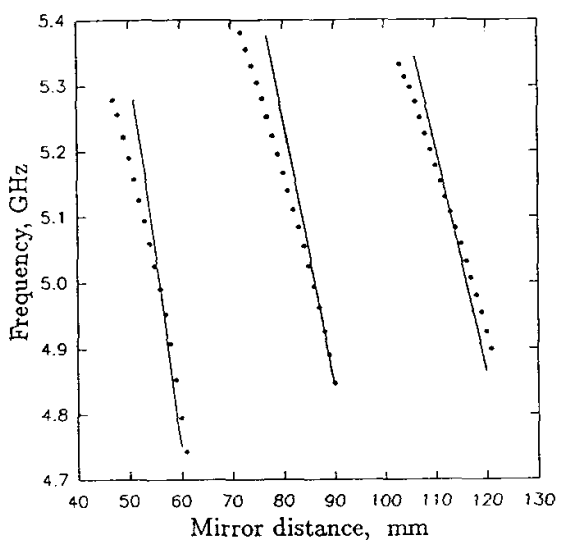

(a)

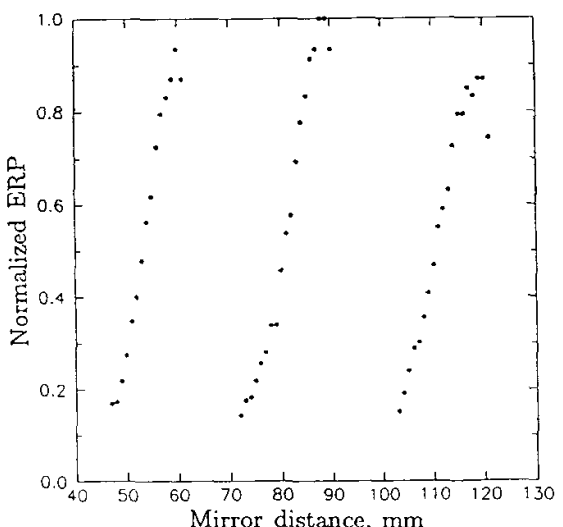

(b)

Fig. 12. (a) Theoretical $(-)$ and measured ( $\cdots$ ) frequency tuning with mirror position. (b) Nomalized ERP as a function of mirror position. The theoretical curve is obtained using the manufacturet's MESFET model with the grid embedding circuit. The mirror position is defined as the distance from the mirror to the back of the grid substrate [36].

of Fig. 9(b) overcomes this problem [37]. Bond wire is used to connect the gate to the horizontal lead. This allows the feedback between the radiated field and the gate to occur through the grid embedding circuit. Figure 13 shows a 16-element gate-feedback grid designed for operation in $\mathrm{X}$-band. The substrate is Rogers Duroid with $\epsilon_{r}=2.2$ and thickness of $2.5 \mathrm{~mm}$. Chip MESFET's, spaced $9 \mathrm{~mm}$ apart, are soldered and wire-bonded to the grid. The width of the device leads are $1 \mathrm{~mm}$. Figure 14 shows the far-field radiation pattern of the grid when a drain bias of $4 \mathrm{~V}$ is applied. The grid oscillates at $11.6 \mathrm{GHz}$ (within $2 \%$ of the design frequency) and produces $335 \mathrm{~mW}$ of output power, corresponding to a dc-to-RF efficiency of $20 \%$.

Illustrating that planar grids can be scaled for use at higher frequencies, the X-band gate-feedback grid was 


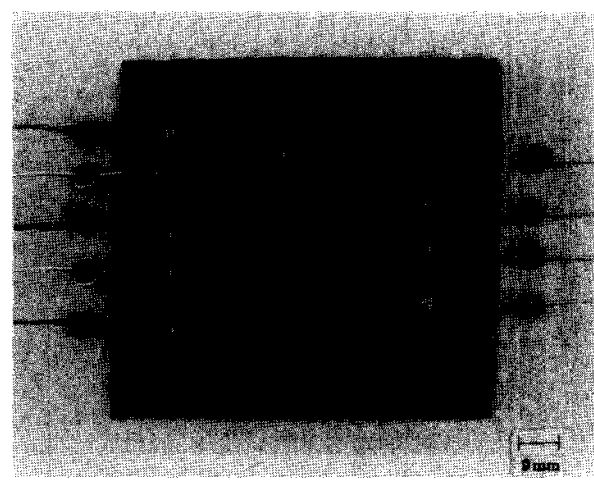

Fig. 13. Photograph of the $\mathrm{X}$-band gate-feedback grid. The substrate is $2.5 \mathrm{~mm}$ thick Rogers Duroid with $\epsilon_{r}=2.2$. The grid is placed between a planar mirror and dielectric slab which form the Fabry-Perot cavity [37].

redesigned for operation in the Ku-band. The scaled grid has $0.5 \mathrm{~mm}$ wide lines and the devices are placed $5 \mathrm{~mm}$ apart. Using an identical substrate and the same devices (FSC11X), a 36-element grid produced $235 \mathrm{~mW}$ at 17 $\mathrm{GHz}$ [37]. This oscillation frequency is near the $f_{\mathrm{T}}$ of the transistors $(19 \mathrm{GHz})$, suggesting that suitably designed HEMT grids may oscillate well over $100 \mathrm{GHz}$. The dcto-RF conversion efficiency of the Ku-band grid is about $7 \%$. This observed reduction in output power and efficiency, compared with the $\mathrm{X}$-band grid, is expected from the higher operating frequency.

\section{AMPLIFIER GRIDS}

Although they are important for many millimeter- and submillimeter-wave systems, oscillators represent only one possible use for MESFET grids. Amplifiers - which are difficult to build at millimeter- and submillimeter-wave frequencies - are necessary in most applications. Grid amplifiers offer the same advantages as grid oscillators: increased power-handling capability and elimination of the losses associated with waveguides and feed networks. In essence, a grid amplifier is a planar structure that radiates an amplified version of a wave incident on its surface. As a result, the grid design must accommodate an array of transistors and suppress potential spurious oscillations. In addition, there needs to be a means of isolating the output wave from the input wave.

Figure 15 shows a unit cell of the grid amplifier. The grid receives radiation polarized in the $y$ direction and radiates a horizontally polarized wave [38]. Polarizing plates are placed on either side of the grid to provide isolation between the amplifier input and output. The unit cell of the grid contains a pair of MESFET's with joined sources. Vertical metal leads are attached to the MESFET gates and horizontal leads connect to the drains. A Duroid substrate with $\epsilon_{r}=10.5$ supports the array. A dc bias, which is applied to lines running horizontally across the back of the substrate, is fed to the MESFET's through via holes.

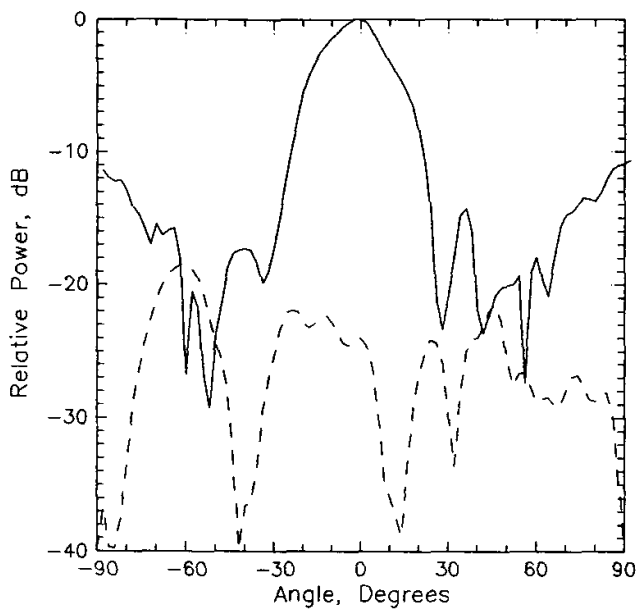

(a)

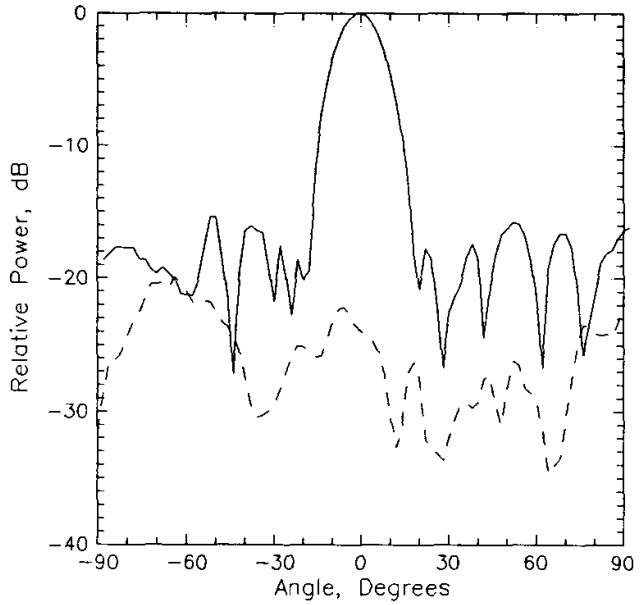

(b)

Fig. 14. Measured far-field radiation patterns for the $\mathrm{X}$-band grid in (a) the $H$ plane (a) and (b) the $E$ plane. The cross-polarized patterns are shown with dashed lines [37]

To eliminate spurious oscillations, $1-\mathrm{k} \Omega$ carbon resistors are placed between the bias lines and the MESFET gates. Additional $120-\Omega$ resistors connect the source leads to $\mathrm{dc}$ ground.

Amplifier gain is measured by illuminating the grid with vertically polarized radiation and measuring the power radiated in the orthogonal polarization. We can write an expression for the amplifier gain as

$$
G=\frac{P_{r}}{P_{c}}\left(\frac{\lambda r}{2 A}\right),^{2}
$$

where $P_{r}$ is the received power with the amplifier grid 


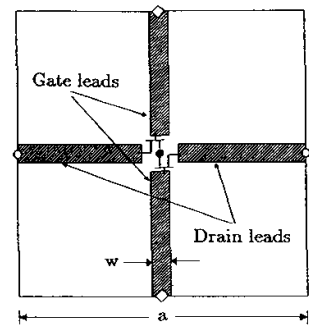

(a)

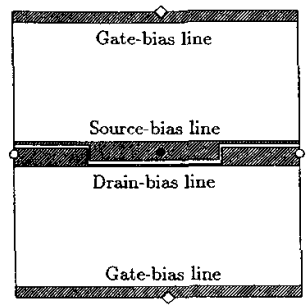

(b)
Fig. 15. A unit cell of the grid amplifier: (a) front view and (b) back view. The symbols indicate different connections between the front and back of the grid amplifier. $\bullet: 120-\Omega$ source-bias resistor; $0: 1-k \Omega$ gate-bias resistor; o: drain-bias pin [38].

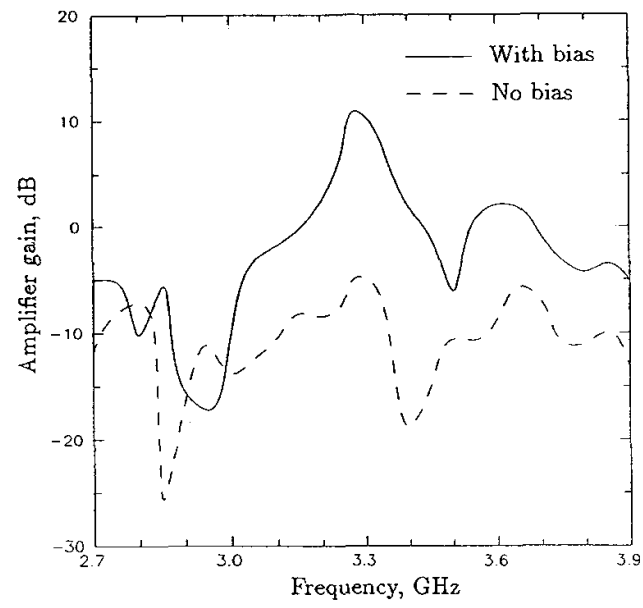

Fig. 16. Measured amplifier gain ( - ) and grid response with no bias(- - ) [38].

in place, $P_{c}$ is the received power with the amplifier grid removed, $A$ is the geometrical area of the grid, and $r$ is the distance between the grid and each horn [38]. This simple formula allows us to calculate the gain from a relative power measurement and three well-known parameters.

Figure 16 shows the measured gain of a 50 -element MESFET amplifier grid. A maximum gain of $11 \mathrm{~dB}$ occurs at $3.3 \mathrm{GHz}$. The input and output polarizers, which are probably responsible for the narrow $(90 \mathrm{MHz}$ ) bandwidth are important; gain is not observed without them. For comparison, the grid response when dc bias is removed is also shown in Fig. 16.

\section{QUASI-OPTICAL SYSTEMS}

Amplifiers and oscillators are just two of the components used in millimeter- and submillimeter-wave systems Many other important system components, such as mixers multipliers, filters, and phase shifters, can also be realized with quasi-optical grids. A common property of these grids

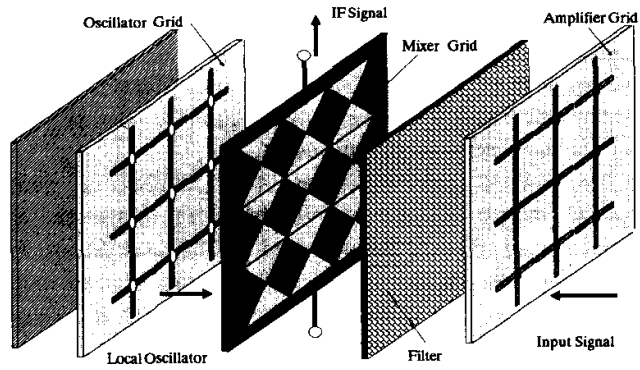

Fig. 17. Schematic of a quasi-optical heterodyne receiver. A grid oscillator provides the $\mathrm{LO}$ and a grid amplifier constimtes the receiver's front end. The IF signal is removed from the mixer with a coaxial cable.

is that their dynamic range increases in proportion to the number of devices in the array, yet noise performance is no worse than for circuits containing a single device.

Lam et al. built a monolithic Schottky-diode grid phase shifter which exhibited $70^{\circ}$ of phase shift at $93 \mathrm{GHz}$ [29]. The same grid, used as a millimeter-wave diode-grid frequency doubler, generated $0.5 \mathrm{~W}$ with $9.5 \%$ efficiency at the second harmonic $(66 \mathrm{GHz}$ ) [30]. More recently, Hacker et al. demonstrated a Schottky diode grid mixer at $10 \mathrm{GHz}$ [39]. The local oscillator (LO) and RF signals illuminate the mixer grid, and the IF signal is removed from the bias lines with a coaxial cable. The grid, which consists of 100 beamlead Schottky diodes in a bow-tie antenna array, has shown a $20 \mathrm{~dB}$ increase in dynamic range over a single diode mixer. The conversion loss and noise figure are comparable to those of a conventional single-diode mixer.

To form a complete quasi-optical system, individual grid components need to be integrated. A heterodyne receiver, for example, consists of a mixer, a local oscillator, an amplifier, and filters. Fig. 17 illustrates how a quasi-optical heterodyne receiver may be realized by cascading grids. An oscillator grid illuminates the mixer to provide an LO. A grid amplifier, followed by a quasi-optical filter, is placed at the receiver input. Dielectric slabs can be included to provide input and output matching. Such a system is straightforward to build and does not require separate antennas that feed waveguide or transmission-line circuits; all signal propagation occurs in free space.

\section{CONCLUSIONS}

This paper has reviewed a variety of MESFET grids used for the generation and amplification of microwave power. The approach, which involves a periodic grid of transistors, is relatively simple to implement and suitable for waferscale integration. Bar grids, which have excellent heatsinking capacity, offer an attractive means of combining the output power of low-efficiency devices such as Gunn diodes. Planar grids, although less efficient at removing heat, are compatible with modern IC fabrication techniques. This is a real advantage for large-scale power combining at millimeter- and submillimeter-wave frequencies. Ex- 
perimental results have shown that the power-handling capacity of quasi-optical grids increases in proportion to the number of devices. Other important figures of merit such as conversion loss and noise figure are no worse than for circuits containing one device.

Much work, however, remains to be done on quasioptical arrays. Questions involving grid robustness and tolerance to device failure need to be addressed. The thermal properties of planar transistor grids require investigation as well as other aspects pertaining to monolithic integration. The operating frequency and output power of oscillator grids must be increased. Better understanding of the nonlinear dynamics of and interaction between devices in the grid may lead to higher dc-to-RF efficiencies. Grid amplifiers are still in their infancy; new grid configurations allowing broader bandwidths and higher-frequency operation need to be studied. In addition, the various issues involved in integrating grid components to form quasioptical systems will need to be examined.

Nevertheless, with the recent advances in IC fabrication technology and the continuing development of new millimeter- and submillimeter-wave devices, quasi-optical power combining remains a most promising method for realizing high output power from solid-state sources.

\section{REFERENCES}

[1] N. C. Luhmann, Jr., "Intrumentation and techniques for plasma diagnostics: An overview," in Infrared and Millimeter Waves, vol. 2, K. J. Button, Ed. New York: Academic Press,1979, ch. 1 .

[2] T. G. Phillips and D. B. Rutledge, "Superconducting tunnel detectors in radio astronomy," Scientific American vol. 254, pp. 96-102, 1986.

[3] J. C. Wiltse, "Introduction and overview of millimeter waves," Infrared and Millimeter Waves, vol. 4, K. J. Button, Ed. New York: Academic Press, 1981, ch. 1.

[4] G. Kantorowicz and P. Palluel, "Backward wave oscillators," in Infrared and Millimeter Waves, vol. 1, K. J. Button, Ed. New York: Academic Press, 1979, ch. 4.

[5] H. Bierman, "Microwave tube design efforts yield steady performance improvements," Microwave J., pp. 52-73, June 1988.

[6] J. W. Hansen, "US TWT's from 1 to $100 \mathrm{GHz}$," Microwave J., pp. 179-193, 1989 (State of the Art Reference).

[7] T. A. DeTemple. "Pulsed optically pumped far infrared lasers," in Infrared and Millimeter Waves, vol. 1, K. J. Button, Ed. New York: Academic Press, 1979, ch. 3.

[8] Y. C. Shih and H. J. Kunno, "Solid-state sources from 1 to $100 \mathrm{GHz}$," Microwave J., pp. 145-161, 1989 (State of the Art Reference).

[9] T. B. Ramachandran, "Gallium arsenide power sources," Microwave J., pp. 91-107, 1990 (State of the Art Reference).

[10] E. R. Brown, T. C. L. G. Sollner, C. D. Parker, W. D. Goodhue, and C. L. Chen, "Oscillations up to $420 \mathrm{GHz}$ in GaAs/AlAs resonant-tunneling diodes," Appl. Phys. Lett., vol. 49, pp. 1777-1779, Oct. 1989

[11] S.P Benz and C. J. Burroughs, "Coherent emission from twodimensional Josephson junction arrays," Appl. Phys. Lett., vol. 58, pp. 2162-2164, May 1991

[12] M. Y. Kao et al., "Very high power-added efficiency and low-noise 0.15- $\mu \mathrm{m}$ gate-length pseudomorphic HEMT's," IEEE Electron Device Lett., vol. 10, pp. 580-582, Dec. 1989.

[13] C. A. Liechti, "High-speed transistors: Directions for the 1990's," Microwave J. pp. 165-177, 1989 (State of the Art Reference).

[14] J. A. Higgins, "GaAs heterojunction bipolar transistors: A second generation microwave power amplifier transistor," $\mathrm{Mi}$ crowave J., pp. 176-194, May 1991.
[15] K. Chang and C. Sun, "Millimeter-wave power combining techniques," IEEE Trans. Microwave Theory Tech., vol. 31, pp. 91-107, Feb. 1983.

[16] K. Kurokawa, "The single-cavity multiple-device oscillator," IEEE Trans. Microwave Theory Tech., vol. 19, pp. 793-801, Oct. 1971.

[17] J. W. Mink, "Quasi-optical power combining of solid-state millimeter-wave sources," IEEE Trans. Microwave Theory millimeter-wave sources," IEEE Trans.
Tech., vol. 34, pp. 273-279, Feb. 1986.

[18] L. Wandinger and V. Nalbandian, "Millimeter-wave power combiner using quasi-optical techniques," IEEE Trans. Microwave Theory Tech., vol. 31, pp. 189-193, Feb. 1983.

[19] K. D. Stephan, S. L. Young, and S. C. Wong, "Microstrip circuit applications of high $Q$ open microwave resonators," IEEE Trans. Microwave Theory Tech., vol. 36, pp. 1319-1327, Sept. 1988.

[20] S. L. Young and K. D. Stephan, "Stabilization and power combining of planar microwave oscillators with an open resonator," in 1987 IEEE MTT-S Int. Symp. Dig. (Las Vegas, NV) vol. 1 , pp. $185-188$.

[21] R. A. York and R. C. Compton, "Quasi-optical power combining using mutually synchronized oscillator arrays," IEEE Trans. Microwave Theory Tech., vol. 39, pp. 1000-1009, June 1991.

[22] K. Chang, K. A. Hummer, and J. L. Klein, "Experiments on injection locking of active antenna elements for active phased arrays and spatial power combiners," IEEE Trans. Microwave Theory Tech., vol. 37, pp. 1078-1084, July 1989 .

[23] A. Mortazawi and T. Itoh, "A periodic second harmonic spatial power combining oscillator," in 1990 IEEE MTT-S Int. Symp. Dig. (Dallas, TX), vol. 3, pp. 1213-1216.

[24] J. Birkeland and T. Itoh, "A 16-element quasi-optical fet oscillator power-combining array with external injection locking," IEEE Trans. Microwave Theory Tech., vol. 40, pp. 475-481, Mar. 1992.

[25] J. A. Annaud and A. Pelow, "Resonant-grid quasi-optical diplexers," Bell Syst. Tech. J., vol 54, pp. 263-283, 1975.

[26] R. Watanabe, "A novel polarization independent beam splitter," IEEE Trans. Microwave Theory Tech., vol. 8, pp. 685-689, 1980.

[27] R. Ulrich, T. J. Bridges, and M. A. Pollack, "Variable metal mesh coupler for far infrared lasers," Appl. Opt., vol. 11, pp. 2511-2516, 1970.

[28] D. B. Rutledge and S. E. Schwarz, "Planar-multimode detector arrays for infrared and millimeter-wave applications," IEEE J. Quantum Electron," vol. 17, pp. 407-414, Mar. 1981.

[29] W. W. Lam et al., "Millimeter-wave diode-grid phase shifters," IEEE Trans. Microwave Theory Tech., vol. 36, pp. 902-907, May 1988.

[30] C. F. Jou et al., "Millimeter-wave diode-grid frequency doubler," IEEE Trans. Microwave Theory Tech., vol. 36, pp. 1507-1514, Nov. 1988.

[31] Z. B. Popović, M. Kim, and D. B. Rutledge, "Grid oscillators," Int. J. Infrared and Millimeter Waves, vol. 9, no. 7, pp. 647-654, 1988.

[32] Z. B. Popović, R. M. Weikle, II, M. Kim, K. A. Potter, and D. B. Rutledge, "Bar-grid oscillators," IEEE Trans. Microwave Theory Tech., vol. 38, pp. 225-230, Mar. 1990.

[33] M. Nakayama. M. Hieda, T. Tanaka, and K. Mizuno, "Millimeter and submillimeter wave quasi-optical oscillator with multi-elements," in 1990 IEEE MTT-S Int. Symp. Dig. (Dallas, TX), vol. 3, pp. 1209-1212.

[34] R. L. Eisenhart and P. J. Khan, "Theoretical and experimental analysis of a waveguide mounting structure," IEEE Trans. Microwave Theory Tech., vol. 19, pp. 706-719, Aug. 1971.

[35] R. E. Ziemer and W. H. Tranter, Principles of Communications, 2nd ed. Boston: Houghton Mifflin, 1985, pp. 146-148.

[36] Z. B. Popović, R. M. Weikle II, M. Kim, and D. B. Rutledge, "A 100-MESFET planar grid oscillator," IEEE Trans. Microwave Theory Tech., vol. 39, pp. 193-200, Feb. 1991.

[37] R. M. Weikle, II, M. Kim, J. B. Hacker, M. P. De Lisio, and D. B. Rutledge, "Planar MESFET grid oscillators using gate feedback," IEEE Trans. Microwave Theory Tech., vol. 40, Nov. 1992.

[38] M. Kim et al., "A grid amplifier," IEEE Microwave and Guided Wave Letters, vol. 1, pp. 322-324, Nov. 1991.

[39] J. B. Hacker, R. M. Weikle Il, M. Kim, M. P. De Lisio, and D. B. Ruttedge, "A 100-element planar Schottky diode grid mixer," IEEE Trans. Microwave Theory Tech., vol. 40, Mar. 1992.

PROCEEDINGS OF THE IEEE, VOL. 80, NO. 11, NOVEMBER 1992 


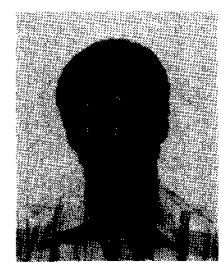

Robert M. Weikle II (Member, IEEE) was born in Tacoma, WA, on February 13, 1963. He received the B.S. degree in electrical engineerin and physics from Rice University, Houston, TX in 1986 and the M.S. and Ph.D. degrees from the 1992 .

, respectively

He is currently with the Department of Applied Electron Physics at Chalmers University of Technology, Göteborg, Sweden. His research interests include microwave and millimeter-wave solid-state devices, high-frequency circuit design, and quasi-optical components and techniques.

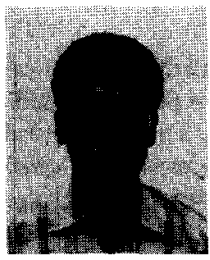

Moonil Kim (Student Member, IEEE) was born in Seoul, Korea, on March 14, 1965. He received the B.S. degree from the Illinois Institute of Technology in 1987 and the M.S. degree from the California Institute of Technology in 1988. the California Institute of Technology in 1988. He is currently working t Caltech. His research interests include quasi-optical millimeter power combining components such as grid oscillators and amplifiers.

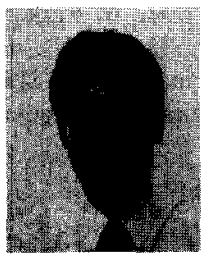

Jonathan B. Hacker (Student Member, IEEE) was born in Vancouver, British Columbia, Canada, on February 16, 1963. He received the B.A.Sc. degree in electrical engineering in 1986 from the University of British Columbia, Vancouver, Canada, and the M.S. degree in electrical engineering from the Califomin Institute of Technology, Pasadena, in 1990 . He is currently degree in electrical engineering at Caltech. From 1986 to 1988 he was associated with the communications research and development group of the Nexus Engineering Corporation. His research intersts include millimeter-wave quasi-optical techniques, microwave power amplifiers, and computer-aided design and measurement of microwave circuits.

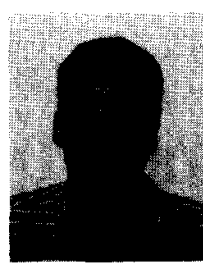

Michael P. De Lisio (Student Member, IEEE) was born in Detroit, MI, on July 29, 1968. He received the B.S.E. degree in electrical engineering from the University of Michigan, Ann Arbor, in 1990. In 1991, he obtained the M.S. degree from the California Institute of Teching nology. Monich methods of millimeter wave power combining and high-frequency solid-state devices are among his research interests. $H$ is currently pursuing the Ph.D. degree at the California Institute of Technology.

Mr. De Lisio is a member of Tau Beta Pi and Eta Kappa Nu.

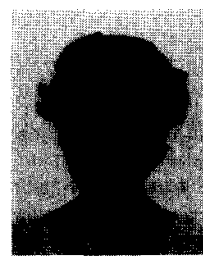

Zoya Basta Popović (Member, IEEE) was born in Belgrade, Yugoslavia, in 1962. She received the Dipl.Ing. degree from the University of Belgrade, Yugoslavia, in 1985 and the M.S. and Ph.D. degrees from the California Institute of Technology, Pasadena, in 1986 and 1990 respectively. Her doctoral thesis was on largescale quasi-optical microwave power combining.

In August 1990 she joined the faculty at the University of Colorado, Boulder, as an Assistant Professor of Electrical Engineering. Her research interests include millimeter-wave quasi-optical techniques, microwave and millimeter-wave active antennas and cjscuits, and optical control of microwave devices.

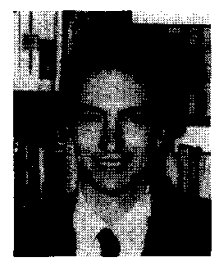

David B. Rutledge (Senior Member, IEEE) was bom in Savannah, GA, on January 12, 1952. He received the B.A. degree in mathematics from Williams College, Williamstown, MA, in 1973 the M.A. degree in electrical sciences from Cambridge University, Cambridge, England, in 1975, and the Ph.D. degree in electrical engineering from the University of California at Berkeley in 1980.

In 1980 he joined the faculty at the California Institute of Technology, Pasadena, where he is now Professor of Electrical Engineering. His research is in developin millimeter- and submillimeter-wave monolithic integrated circuits and applications and in software for computer-aided design and measurement. Dr. Rutledge is coauthor of the software CAD program Puff, which has 8000 users worldwide. 